\title{
PRADER-WILLI SYNDROME
}

The clinical and polysomnographic features of 5 patients with PraderWilli syndrome were studied at the Mayo Clinic, Jacksonville, FL. All patients, including 2 adults, 1 adolescent, and 2 children were obese; they had hypersomnolence and daytime hypoxemia, and they snored. The apnea plus hyponea index was less than 10 episodes per hour of sleep. Polysomnography failed to demonstrate clinically severe obstructive apnea or hypopnea. Sleep architecture and rapid eye movement latency were normal in all patients. Nonapneic reductions in oxyhemoglobin saturation were detected in only 1 adult and in 1 child. Despite the presence of morbid obesity and a history of snoring these patients with Prader-Willi syndrome had only mild sleepdisordered breathing (Kaplan J et al. Sleep and breathing in patients with the Prader-Willi syndrome. Mayo Clin Proc Nov 1991; 66:1124-1126).

COMMENT. Prader-Willi syndrome is characterized by infantile hypotonia, early childhood obesity, mental deficiency, short stature, small hands and feet and hypogonadism. Many patients also have hypersomnolence, daytime hypoventilation, and some die prematurely of cardiorespiratory failure. The epidemiology of Prader-Willi syndrome was studied in a rural county in Sweden and reported from the Department of Pediatrics and Child Psychiatry, Child Neuropsychiatry Centre, Goteborg, Sweden (Akefeldt A et al. Dev Med

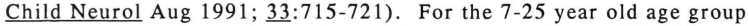
the prevalence of Prader-Willi syndrome was 13 per 100,000 for definite cases, and 20 per 100,000 if borderline cases were included.

Anesthesia and the Prader-Willi syndrome was the subject of a report from the John Radcliffe Hospital, Oxford, England (Mackenzie

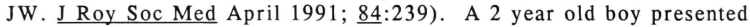
for correction of ventral curvature of micropenis and an 18 year old received anesthetic for removal of excess tissue from one thigh. Halothane in nitrous oxide and oxygen were used for induction and isoflurane to maintain anesthesia. Attention to glycaemic control and body temperature is important since glucose intolerance and both hypothermia and hyperthermia have been reported in response to operative stress.

\section{ACROCALLOSAL SYNDROME}

A large highly inbred kindred with 3 definite and 1 possibly affected siblings with acrocallosal syndrome is reported from the Kaplan Hospital, Rehovot and the Emek Central Hospital, Afula, Israel. The family is of Israeli-Arab extraction and the parents are first cousins. The clinical features are divided into three groups: a) diagnostic/essential findings; b) frequent findings; and c) occasional/associated features. The diagnostic findings include macrocephaly, prominent forehead, hypertelorism, epicanthal folds, downslanted palpebral fissures, small nose with wide nasal bridge, polydactyly and absent/hypoplastic corpus callosum. Frequent findings include ear anomalies, short upper lip, high arched palate, tapered fingers, hypotonia, and 